\title{
Zero dispersion wavelength measurement through soliton self- frequency shift compensation in suspended core fibre
}

B. Kibler, C. Finot, G. Gadret, G. Millot, J. Wojcik, M. Szpulak, W. Urbanczyk

\section{Authors' affiliations:}

B. Kibler, C. Finot, G. Gadret, G Millot

Institut Carnot de Bourgogne, UMR 5209 CNRS-Université de Bourgogne, Dijon, FRANCE.

J. Wojcik

Maria Curie-Skłodowska University, Laboratory of Optical Fiber Technology, Lublin, POLAND.

M. Szpulak, W. Urbanczyk

Wroclaw University of Technology, Institute of Physics, POLAND.

Contact Author: B. Kibler

Contact Address: Institut Carnot de Bourgogne, CNRS UMR 5209

9 Avenue Alain Savary, BP 47870

21078 Dijon

FRANCE

Fax: $\quad$ +33 (0)380 $\quad$ (295971

Phone: $\quad+33(0) 380395932$

Email:_Bertrand.Kibler@u-bourgogne.fr 


\begin{abstract}
We describe a simple experimental technique to evaluate the second zero dispersion wavelength of very small core microstrutured fibres. Based on the effect of soliton selffrequency shift and its subsequent compensation in the vicinity of the second zero dispersion, the proposed method is applied to both standard and suspended core microstructured fibres by simply measuring the frequency stabilized soliton spectrum, avoiding any calculation and the knowledge of any experimental parameters.
\end{abstract}


Since their first developments in the past decade, microstructured fibres have motivated major progresses in nonlinear guided optics by offering an outstanding flexibility in the design of the dispersive and nonlinear properties [1]. Suspended core fibres are a new example of the extreme light confinement that is feasible in photonic crystal fibres with sub-micron core diameters [2]. This tight light confinement significantly affects the dispersion characteristics, leading to a local extremum in the group-velocity dispersion (GVD) curve and a second zero dispersion wavelength (IR-ZDW) below $2 \mu \mathrm{m}$. Several powerful fully vectorial techniques have been proposed to simulate the wavelengthdependent parameters of the guided modes, such as dispersion and effective area. Real microstrutured fibre structures can be taken into account through the SEM image of their fibre cross section [3]. However, the finite accuracy of the edge detection holes and the SEM image quality itself can induce some discrepancies between caculated and real fibre parameters, especially in the prediction of the IR-ZDW which is very sensitive to the fibre geometry [4]. It is therefore of high interest to benefit from an easy-to-implement experimental method to check for IR-ZDW value. In the present work, we propose such a method based on the shift and control of the central wavelength of an ultrashort pulse, in the context of small core microstructured fibres. With the help of experimental results obtained in commercial PCF with two ZDWs, we illustrate two major spectral dynamics of solitons, i.e. their self-frequency shift and their frequency stabilization. From comparison with numerical simulations, we are able to present and standardize a simple experimental method to determine the IR-ZDW, by simply measuring the frequency stabilized soliton spectrum. We also apply our technique to a suspended core fibre and experimental results are confirmed by numerical calculations of GVD curve.

First, we have investigated specific spectral dynamics of solitons in the anomalous dispersion regime of our PCF exhibiting two ZDWs, with a simple experimental setup as shown in Fig.1. The source is a Ti:Sapphire laser (Mira Coherent) delivering 160-fs FWHM pulses at $891 \mathrm{~nm}$. The use of a conventional half-wave plate-polarizer allow us to modify the input power while keeping the input linear polarization parallel to one of the 
PCF birefringent axes. This scenario can lead to spectral broadening over several hundred of $\mathrm{THz}$, for input pulse energies sufficient to stimulate higher order solitons dynamics [5]. The evolution of experimental spectra recorded for various input pulse energy (Fig.2a) highlights two major events. First, the major process of spectral broadening is Raman scattering, resulting in a continuous frequency shift of solitons emitted to higher wavelengths. Secondly, once the shifted soliton reached the vicinity of the IR-ZDW, we can also observe its frequency stabilization accompanied by the generation of new spectral components. Indeed, for this PCF, the solitons stabilize around $1190 \mathrm{~nm}$ (near the IR-ZDW given by the manufacturer at $1235 \mathrm{~nm}$ ), and new spectral components called dispersive waves are generated around $1350 \mathrm{~nm}$ in the normal dispersion regime when a resonance condition can be satisfied. This coupling between soliton and wave dispersive leads to a spectral recoil of the soliton to shorter wavelengths, which compensates the Raman shift towards longer wavelengths [6].

The experimental dynamics is compared to numerical simulations of the nonlinear propagation in our 2-m segment of PCF. A generalized nonlinear Schrödinger equation approach taking into account the global dispersion characteristics and nonlinearity provided by the manufacturer is used [5]. Numerical results are shown in Fig.2b, soliton frequency shifts (from the pump frequency) are shown for the first two solitons (S1-S2). Then, we clearly confirm both distinct spectral dynamics, firstly a frequency shift from 891 to $1210 \mathrm{~nm}$ for input energies below $100 \mathrm{pJ}$ (for S1), and secondly an interruption of this shift leading to a frequency stabilization for higher energies. The soliton S2 also shows similar dynamics. Experimental observations of both SSFS and stabilization processes are in good agreement with numerical results. However, we can note a slight difference about $20 \mathrm{~nm}$ between the final stabilization wavelengths obtained numerically and experimentally, as it is also shown in Fig.2c. As the stabilization frequency does not depend on the input power or initial pulse shape, we can infer that this observed difference is related to discrepancies between the GVD curve provided by the manufacturer and the real PCF segment dispersion, in particular in the IR-ZDW region. Assuming that the real dispersion curve is simply red-shifted, it is possible to estimate the 
second ZDW around $1245 \mathrm{~nm}$ (see Fig.2c). In order to verify the robustness of our proposed estimate, we have performed additional numerical simulations of soliton dynamics close to the IR-ZDW, based on a simplified numerical model including the Raman effect and the second and third order dispersion coefficients ( $\beta_{2}$ and $\left.\beta_{3}\right)$. Varying values of $\beta_{2}$ and $\beta_{3}$ about $\pm 100 \%$ while keeping the same IR-ZDW, but also other parameters such as soliton peak power and its temporal width, and the ratio $\beta_{3} / \beta_{2}$, we have studied both variations of the stabilization wavelength and the soliton relative intensity at IR-ZDW. Then, we have infered that the intensity of S1 at IR-ZDW still remains about $10-25 \%$ of its maximum, corresponding here to an error around $\pm 10 \mathrm{~nm}$ in the position of the IR-ZDW. The central wavelength of soliton stabilization varies more significantly and cannot be used as the only reference point to determine the IR-ZDW.

Now, we put into practice our simple technique to determine the second ZDW of a 20-mlong suspended core fibre manufactured at Maria Curie-Sklodowska University, exhibiting a small silica core diameter estimated to $0.96 \mu \mathrm{m}$ (Fig.3a). We used a fully vectorial mode solver based on Finite Element Method in order to calculate dispersion characteristics of the fundamental mode (Fig.3c) [7]. SEM image of the fibre cross-section was numerically treated to accurately reproduce the image of the real fibre cross-section and calculations were based on a mesh composed of about 100000 elements. The two ZDWs have been estimated to 620 and $1169 \mathrm{~nm}$. To evaluate the impact of slight variations (around ten $\mathrm{nm}$ ) of the core size which may arise from edge detections errors or from longitudinal fluctuations in the fabrication process [1], we have performed other dispersion calculations, taking into account variations of core diameter up to $\pm 2 \%$ (see Fig. $3 c$ ). The high sensitivity of the IR-ZDW is numerically confirmed with variations as high as $\pm 25 \mathrm{~nm}$ in the position of the IR-ZDW. The experimental setup used is similar as in Fig.1, but the input wavelength is now $832 \mathrm{~nm}$. Fig.3b shows the experimental spectra obtained for increasing input power. We observe the self-frequency shift of various fundamental solitons until they reach the stabilization wavelength around $1144 \mathrm{~nm}$, in the vicinity of the IR-ZDW. Following our previous analysis, we can estimate the IR-ZDW assuming the 
intensity of stabilizated soliton at ZDW close to $10-25 \%$ of its maximum intensity. This method gives us the IR-ZDW at $1152 \pm 5 \mathrm{~nm}$ (see Fig.3c). The accuracy is here better due to the narrow soliton spectrum observed. Our experimental value for the IR-ZDW is fully consistent with numerical results. Similarly to our previous analysis, we have verified here that both fibre birefringence and inversion of fibre extremities do not modify these results more than their accuracy. Let us specify that our method gives the ZDW value for the last centimeters of the fibre segment. Indeed, in case that the stabilized soliton undergoes possible longitudinal fluctuations of GVD, its own spectrum then shifts in frequency to follow these variations.

In conclusion, by simply measuring the frequency stabilization spectrum of the soliton in the vicinity of the IR-ZDW, we accurately evaluate this sensitive ZDW, without exactly knowing any experimental parameters. This simple experimental method only requires ps or fs pulse sources whose the frequency is set between the two ZDWs, and an optical spectrum analyzer. We demonstrate a convenient technique to complement numerical dispersion calculations of small-core microstructured fibers.

Acknowledgements: This research was supported by the Agence Nationale de la Recherche (SUPERCODE project, ANR-06-BLAN-0401 ; SOFICARS project, ANR-07RIB-013-03). We also acknowledge support by the POLONIUM project and the COST 299 Action FIDES. 


\section{References}

1. P. St. J. Russell, " Photonic-Crystal Fibers," Journal of Lightwave Technology 24, 4729-4749 (2006).

2. C. M. Cordeiro, M. A. Franco, C. J. Matos, F. Sircilli, V. A. Serrao, C. H. Cruz, "Single-design-parameter microstructured optical fiber for chromatic dispersion tailoring and evanescent field enhancement," Optics Letters 32, 3324-3326 (2007).

3. M. Szpulak, W. Urbanczyk, E. Serebryannikov, A. Zheltikov, A. Hochman, Y. Leviatan, R. Kotynski, K. Panajotov, "Comparison of different methods for rigorous modeling of photonic crystal fibers," Optics Express 14, 5699-5713 (2006).

4. N. Y. Joly, F. G. Omenetto, A. Efimov, A. J. Taylor, J. C. Knight, P. St. J. Russell, "Competition between spectral splitting and Raman frequency shift in negativedispersion slope photonic crystal fiber," Optics Communications 248, 281-285 (2005).

5. J. M. Dudley, G. Genty, S. Coen, "Supercontinuum generation in photonic crystal fiber," Reviews of Modern Physics 78, 1135-1184 (2006).

6. F. Biancalana, D. V. Skryabin, A. V. Yulin, "Theory of the soliton self-frequency shift compensation by the resonant radiation in photonic crystal fibers," Physical Review E 70, 016615 (2004).

7. M. Koshiba, S. Maruyama, K. Hirayama, "A vector finite element method with the higher order mixed-interpolation-type triangular elements for optical waveguide problems," Journal of Lightwave Technology 12, 495-502 (1994). 


\section{Figure Captions:}

Fig. 1 Experimental setup with the commercial PCF parameters provided by the manufacturer.

Fig. 2 (a) Experimental output spectra (linear intensity scale) obtained for various input pulse energy. (b) Comparison of numerical and experimental SSFS (from the pump frequency) for the first two solitons. (c) Numerical and experimental spectra showing the stabilisation wavelength of $\mathrm{S} 1$.

Fig. 3 (a) SEM image of the suspended core fibre with its estimated diameter of the triangular silica core. (b) Experimental spectra (linear intensity scale) obtained for various input pulse energy. The dashed line represents the estimated IRZDW. (c) Numerical dispersion calculations of the fundamental mode of the suspended core fibre performed from the SEM image (solid line), and with slight variations $( \pm 2 \%)$ of the core diameter (dahed lines). The experimental estimate of the IR-ZDW is also shown with its error bar. 


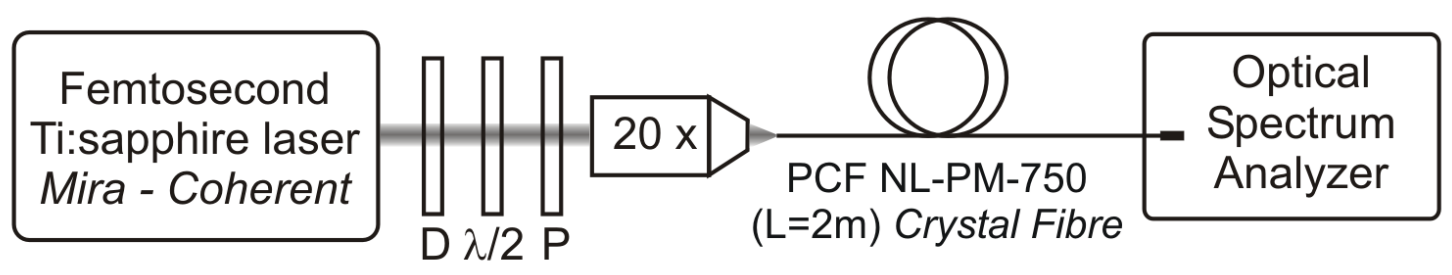

D: neutral density

PCF parameters:

$\lambda / 2$ : half-wave plate

$\mathrm{V}-\mathrm{ZDW} \sim 750 \mathrm{~nm}$

$\mathrm{P}$ : polarizer

IR-ZDW $1235 \mathrm{~nm}$

Loss $\sim 0.05 \mathrm{~dB} / \mathrm{m}$

$\gamma(@ 800 \mathrm{~nm}) \sim 0.95 \mathrm{~W}^{-1} \cdot \mathrm{km}^{-1}$

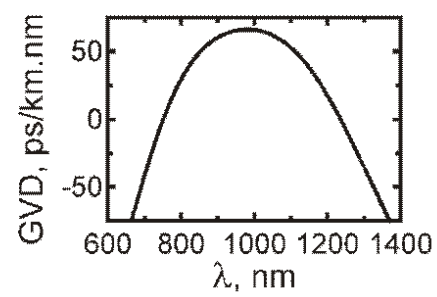

FIGURE 1 

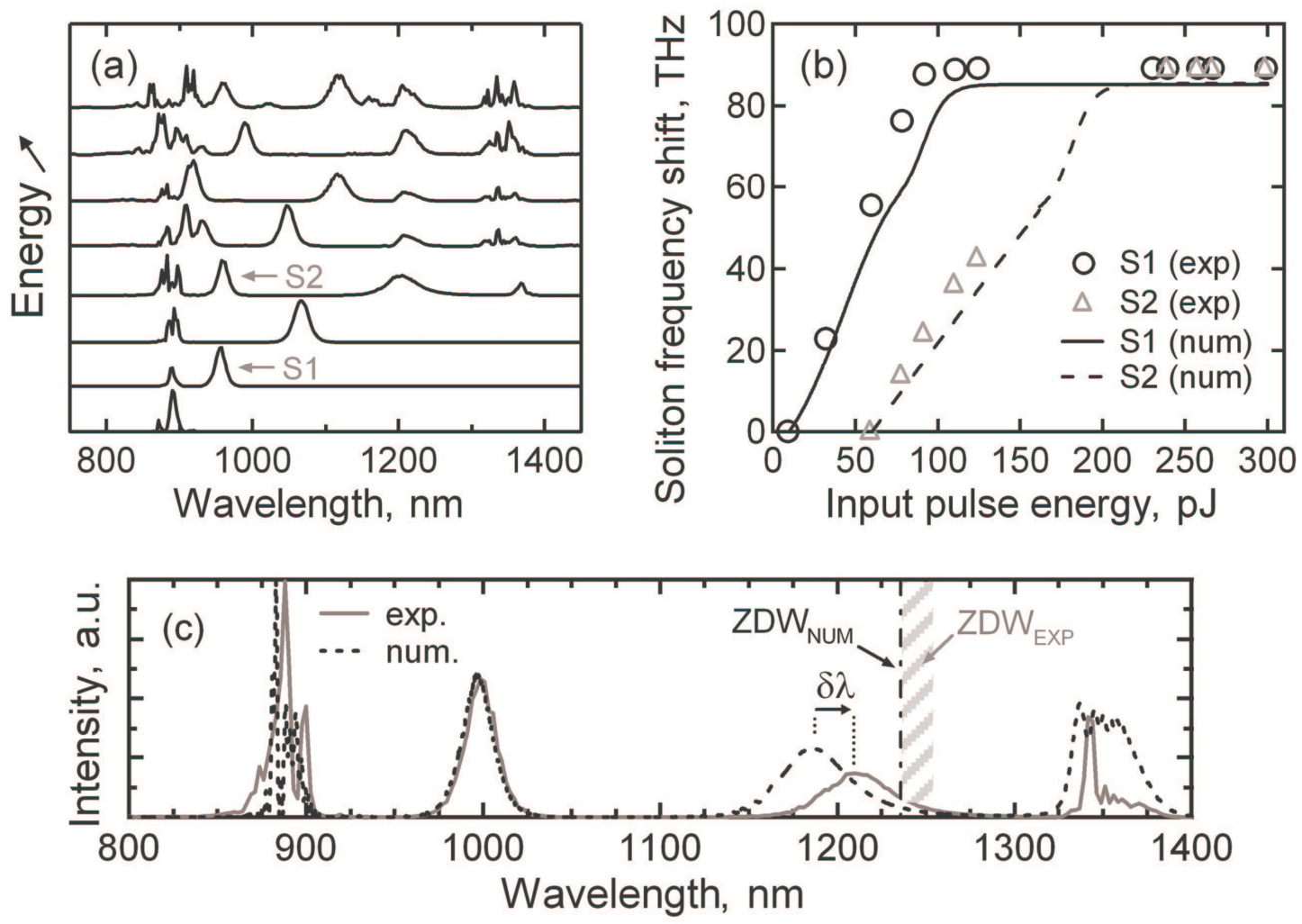

FIGURE 2 

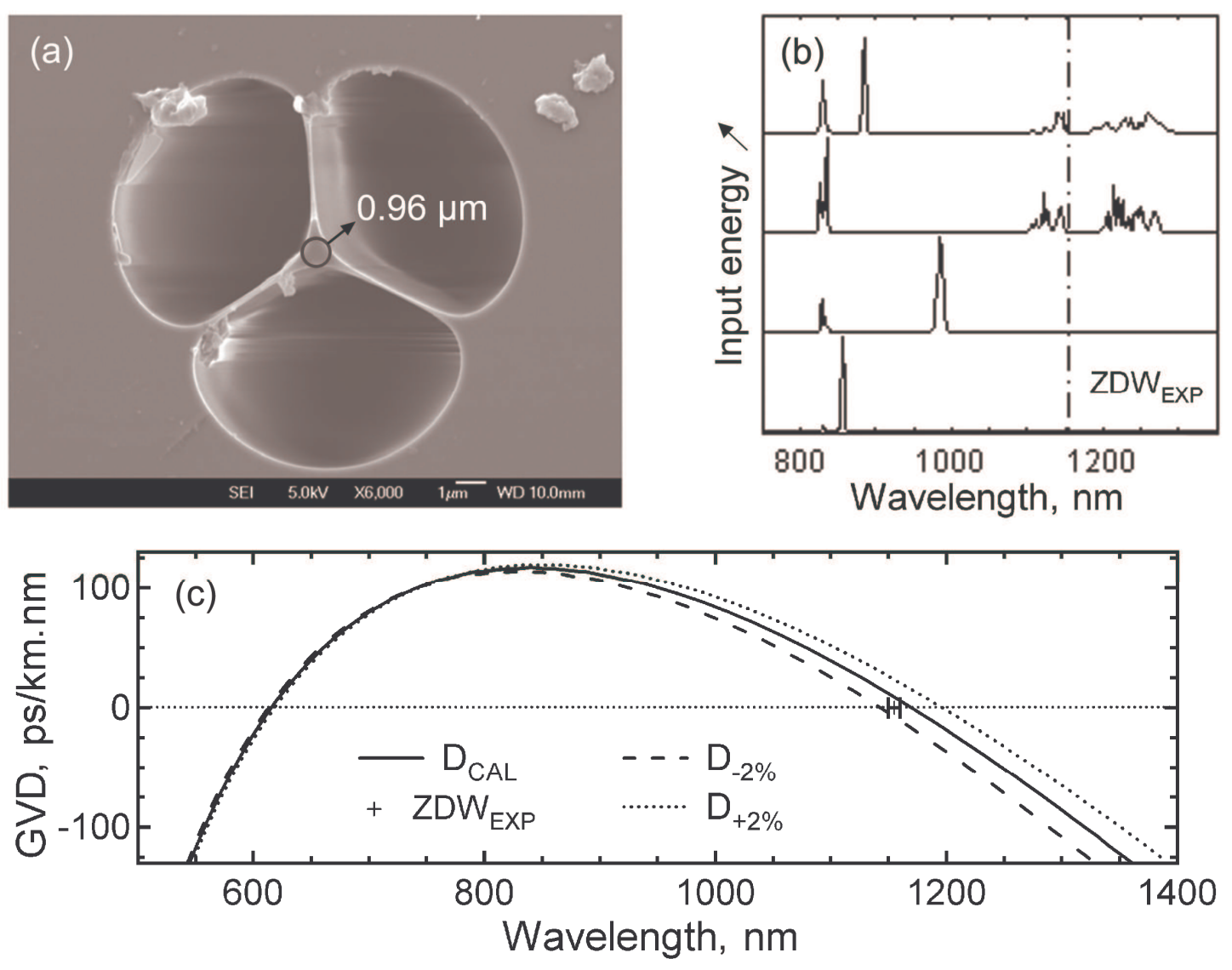

FIGURE 3 